\title{
EL ANAGRAMA SAUSSURIANO. LOS TEXTOS Y LA CRÍTICA ${ }^{1}$
}

\author{
Raúl Rodríguez Ferrándiz
}

\author{
Universidad de Alicante
}

C'est une expérience facile à faire, après avoir donné l'attention nécessaire au détail qui nous rive à l'écriture, de reprendre à haute voix ces groupes, et alors l'oreille dira son mot, et dans la plupart des cas déclarera qu'elle a reçu une impression d'ensemble rappelant en effet le nom ou le mot qui est en cause, et qui domine ordinairement le passage.

\section{INTRODUCCIÓN}

Entre 1906 y 1909, es decir, de forma contemporánea a los dos primeros cursos de lingüística general impartidos en la Universidad de Ginebra, Ferdinand de Saussure investigó ciertas figuras fónicas en

1 Este trabajo resume y reordena parte de los materiales que compusieron nuestra tesis doctoral inédita La semiótica anagramática de Ferdinand de Saussure. Génesis. crítica y tipología, leída en la Universidad de Alicante en abril de 1997. 
varias tradiciones literarias del dominio indoeuropeo, y en particular en la poesía latina, consignando sus descubrimientos en más de cien cuadernos de notas. Nunca se decidió a publicar los resultados, y los manuscritos permanecieron inéditos no sólo a su muerte, en 1913, sino durante medio siglo más. La prolongada desatención hacia estos textos de los editores de Saussure -Bally y después Godel los conocieron bien-concluyó a mediados de la década de los sesenta gracias al celo de Jean Starobinski, quien publicó en varias entregas extractos de los cuadernos, material que fue recogido, ordenado y oportunamente presentado y comentado en el volumen Les mots sous les mots. Les anagrammes de Ferdinand de Saussure (Starobinski, $1971^{2}$ ), verdadera vulgata de un trabajo que aún hoy no ha recibido una edición crítica íntegra. A partir de entonces se sucedieron algunas publicaciones, por lo general breves, de otros fragmentos inéditos procedentes de los cuadernos (Rossi, 1968; Wunderli, 1972a, 1972b; Rey, 1973; Lotringer, 1974; Starobinski, 1974; Ossola, 1979; Shepheard, 1982, 1984; Prosdocimi-Marinetti, 1991), así como de la reveladora correspondencia intelectual de Saussure durante el periodo que nos interesa (Benveniste, 1964; Jakobson, 1971; Minassian, 1976; Amacker, 1995), que demuestra la absorbente dedicación del sabio a la hipótesis del anagrama, el conocimiento detallado que de ella tuvieron alumnos y colegas como Bally y Meillet, y el apoyo que recibió sobre todo de éste último, quien le animó a una inmediata publicación (158-159, Jakobson, 1988:150).

La bibliografía saussuriana, tan corta en el propósito editorial del propio Saussure ${ }^{3}$, se veía así inopinadamente ampliada con los llamados Cahiers d'Anagrammes, de dimensiones y calado tan considerables, a los que habría que sumar la edición de otra serie de cuadernos manuscritos, los Nibelungen (Avalle, 1973; Prosdocimi, 1983; Saussure, 1986), que versan sobre la caracterización semiológica de las leyendas germánicas y que están conectados con aquéllos ${ }^{4}$, así como su juvenil

2 En adelante las páginas entre paréntesis, sin indicación de nombre y fecha, remitirán a este libro.

3 Como es bien sabido, el maestro ginebrino sólo se decidió a publicar dos obras durante su vida, y la última cuando todavía contaba 24 años. Se trata de su Memoire sur le système primitive des voyelles dans les langues indoeuropéennes y De l'emploi du génitif absolu en sanscrit, respectivamente su memoria de licenciatura y su tesis doctoral. Desde entonces nada dio a la imprenta salvo unas pocas publicaciones breves, que ralearon mucho sobre todo a partir de 1893, siendo sólo cinco entre 1900 y 1912.

4 Hemos intentado demostrar este extremo en «El mito del signo y el signo del mito en Saussure y en Lotman». Comunicación presentada en la Reunión Internacional in memoriam Iuri M. Lotman. Granada, 26-28 de octubre de 1995 (en prensa las Actas). 
Essai pour réduire les mots du Grec, du Latin et de l'Allemand à un petit nombre de racines sobre el origen de las lenguas y los estudios indológicos y fonológicos que se conservan en la Universidad de Harvard. La obra completa de Saussure, que nos permitiría estudiar el conjunto y el proceso de su pensamiento filológico, aún no se conoce plenamente y es, por lo tanto, una obra todavía abierta. Ahora bien, la exhumación de estos inéditos de Saussure, y en particular de los Cuadernos de Anagramas que nos van a ocupar aquí, provocaron una verdadera convulsión en el panorama semiolingüístico, sobre todo en Francia, y alimentaron una polémica virulenta en ocasiones, pero fértil en aportaciones de la más variada índole, sobre el legado saussuriano.

Nuestra labor en las páginas que siguen será la perentoria presentación de la hipótesis saussuriana, casi completamente desconocida en medios filológicos hispánicos a pesar de su inmediata repercusión y de las numerosas contribuciones críticas que se ocuparon de presentarla, discutirla y, en algunos casos, aplicarla al análisis textual. Haremos también algunas calas en el panorama crítico que depararon los Anagramas en su momento, y mostraremos que la herencia de estos textos alcanza a algunas recientes estrategias y corrientes semiolingüísticas y críticas.

\section{LA HIPÓTESIS ANAGRAMÁTICA SAUSSURIANA}

El origen de la investigación estuvo en un arduo problema de la métrica latina, el verso saturnio, de complicada sujeción a cualquiera de los patrones métrico-rítmicos conocidos. Saussure creyó descubrir que su clave compositiva era en realidad fónica, y consistía en una total duplicación de los sonidos que lo componían, es decir, una especie de aliteración generalizada, por la que cada elemento fónico singular o monófono ${ }^{5}$ aparecía redoblado antes del fin del verso, lo cual propiciaba sumas totales pares al cabo del mismo (20-26; Benveniste, 1964:109-111). En el caso de producirse algún desajuste, algún monófono no emparejado, estaba prevista su compensación en el verso siguiente, que dejaría precisamente ese monófono sin emparejar para

5 Saussure emplea muy raramente la palabra fonema en los cuadernos y cartas dedicados al anagrama (sólo en 39-40 y Benveniste, 1964:114), prefiriendo hablar de elementos fónicos y, precisando más, de monófonos, dífonos, trífonos o polífonos. 
proveer el ajuste pleno. Saussure practicó análisis satisfactorios tanto de saturnios epigráficos como literarios, y así, en el siguiente verso, que forma parte de una inscripción grabada sobre un sarcófago y concierne al cónsul Lucio Cornelio Escipión (33-34):

\section{Subigit omne Loucanam opsidesque abdoucit ${ }^{6}$}

Saussure establece las siguientes equivalencias fónicas, constatando que «il y a une correspondance de tous les éléments se traduisant pour une exacte «couplaison», c'est-à-dire répétition en nombre pair»:

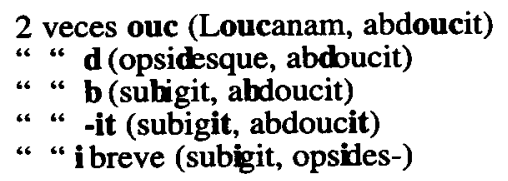

“ “ a breve (Loucaam, abdoucit)

" " o breve (amne, opsides-)

" " $\mathbf{n}$ (omne, Loucanam)

“ " m (omne, Loucanam)

Ciertamente, la simetría en la distribución fónica es grande, pero no total: hay ciertos sonidos que no encuentran su par en el verso, como p (opsidesque). Saussure señala entonces que esos «residuos» hallan su justa correspondencia en los restos que a su vez dejan o el verso precedente o el siguiente. En el caso que nos ocupa, el verso anterior libera una p que restablece la simetría: TAURASIA CISAUNA SAMNIO CErT (33-34).

Saussure decidió aplicar estas leyes del acoplamiento a corpus poéticos de otras tradiciones literarias del dominio indoeuropeo, como la épica homérica (Amacker, 1995:107-113), los himnos védicos (36-38; Shepheard, 1982) y la poesía germánica antigua (38-40, Shepheard, 1984), constatando que en ellos eran no menos implacables. Ahora bien, la ampliación del corpus textual y - como dice Saussure- el «entrenamiento gimnástico» en la atención fónica aplicada sobre los

6 El epitafio consta de una inscripción de cuatro líneas que contiene seis saturnios: [L. Cornelib Cn. f. Scipio: Cornelius Lucius Scipio Barbatus / Gnaivod patre prognatus, fortis vir sapiensque, / Quoius forma virtutei parisuma fuit / Consol censor aidilis quei fuit apud vos, / Taurasia Cisauna Samnio cepit / Subigit omne Loucanam opsidesque abdoucit. La traducción sería «Lucio Cornelio Escipión, hijo de Gneo: Cornelio Lucio Escipión Barbatus, hijo de Gneo su padre, hombre valeroso y sabio cuya belleza igualó a la virtud, que fue entre vosotros cónsul censor edil, tomó Taurasia y Cisauna en el Samnio, somete (sic) toda la Lucania y toma (sic) rehenes». 
versos permitió al maestro afinar el análisis. En primera instancia se produjo una modificación sólo cuantitativa, al percatarse Saussure de que las recurrencias no eran de hecho monofónicas (fonemas sueltos que se repiten a distancia en el interior del verso), sino al menos difónicas (grupos de dos fonemas sucesivos como mínimo, a veces coincidentes con sílabas). Y así, en el siguiente dístico de la Odissia de Livio Andrónico? ${ }^{7}$.

\section{IBI MANENS SEDETO DONIOUM VIDEBIS}

ME CARPENTO VEHENTE DOMUM VENISSE

los grupos polifónicos DE, BI, DO, VE, TO, NI, EN, SE, ENT y UMV aparecen duplicados (35; Benveniste, 1964:110-111). El principio del dífono como unidad irreductible de las recurrencias fónicas tuvo como efecto desactivar la posible objeción de gratuidad de los acoplamientos monofónicos, en buena medida justificables por el limitado inventario de fonemas de cualquier lengua, y establecer un orden mínimo, el de la secuencia difónica. Los análisis practicados nuevamente en versos védicos y homéricos fueron muy satisfactorios, hasta tal punto que en la minuciosa lectura y el recuento de grupos difónicos en estos textos habría de producirse la decisiva inflexión, en este caso cualitativa, de la hipótesis: Saussure descubrió que las insistentes aliteraciones, rimas interiores y acoplamientos fónicos variados tanto en los himnos del Rig-Veda como en los versos de la Ilíada parecían tender a evocar, al sesgo de la lectura poética, un nombre propio, que era invariablemente el del héroe o dios concernido por los versos. $\mathrm{Y}$ así, los nombres Agamemnon, Bellerofontes y Zefiros, descompuestos en sus dífonos, resonaban «anagramáticamente» en el espacio de unos pocos versos homéricos (127; Amacker, 1995:114-119), aquéllos precisamente que versaban sobre ellos, y sin perjuicio de que dichos nombres fueran también enunciados explícita, gramáticamente. En el primer himno del Rig-Veda, Saussure escucha la insistente diseminación de los nombres de las divinidades invocadas en cada estrofa: Agni, Aditya, Indra (37; Shepheard, 1982:519-521). De forma análoga, los versos de las epopeyas germánicas Hildebrandslied y Nibelungenlied anagramatizan los nombres de los héroes -Hiltibrand, Hadubrand, Hagen, Kriemhilt, Sigelint-, y en el Evangelio de Otfrido de Wissemburg (siglo IX), la primera obra de autor conocido escrita en alemán, Saussure descubre la presencia masiva de nombres propios bíblicos que inseminan a cada paso la trama fónica del texto:

7 Luis Alberto de Cuenca y Antonio Alvar traducen el dístico: «Siéntate ahí y espera a que me veas, / conduciendo mi carro, volver a casa». 
Magdalena, Moyses, Samaria, Pilatus, Salomon, Paradyses, Galilea, Barrabas, Kaifas, etc. (Shepheard, 1984:61-64).

La inicial hipótesis fónica de los acoplamientos devino, pues, fonosemántica al venir ordenadas las recurrencias en torno a lo que Saussure llamó palabra-tema, variable semántica novedosa en la teoría pero, como se aprecia, nada críptica o hermética: no se trata de enunciar veladamente, a través de una clave, un nombre censurado $u$ objeto de tabú - como suele ser el caso del anagrama clásico-, sino sancionar, a partir de la propia textura fónica del pasaje, la centralidad temática ya evidente de los protagonistas del relato mítico, la invocación ritual o, andando la investigación, la misma autoría -o el destinatario, o el mecenas, en su caso- en las composiciones de aliento más lírico. Las alusiones de Saussure a este respecto son inequívocas:

L'anagramme peut se dérouler soit sur un nom qui figure dans le texte, soit sur un nom qui n'est pas prononcé du tout, mais se présente naturellement à l'esprit par le contexte (Benveniste 1964:111) Il reste remarquer combien l'anagramme est en irréprochable concordance avec le sens contenu dans les vers, ou la manière dont on les découpera d'après ce sens (Lotringer 1974:105). Les sons se trouvaient faire allusion à un nom que tout le monde avait dans l'esprit (125-126).

Saussure dedujo que esta paráfrasis fónica de un nombre propio era la preocupación que el poeta se imponía constantemente además de la del metro (134), una estrategia textual verdaderamente constrictiva (30), y describió con detalle el procedimiento. El versificador debía

\footnotetext{
Avant tout, se pénétrer des syllabes, et combinaisons phoniques de toute espèce, qui se trouvaient constituer son THEME. Ce thème -choisi par luimême ou fourni par celui qui faisait les frais de l'inscription- n'est composé que de quelques mots, et soit uniquement de noms propres, soit d'un ou deux mots joints à la partie inévitable des noms propres. Le poète doit donc, dans cette première opération, mettre devant soi, en vue de ses vers, le plus grand nombre de fragments phoniques possibles qu'il peut tirer du thème; par exemple, si le thème est Hercolei, il dispose des fragments -lei-, ou -co; ou avec une autre coupe des mots, des fragments -ol-, ou -er-; d'autre part de $r c$ ou de $c l$, etc. Il doit alors composer son morceau en faissant entrer le plus grand nombre possible de ces fragments dans ses vers, par ex. afleicta pour rappeler Herco-lei, ainsi de suite (23-24).

La méthode habituelle et fondamentale du poète consistait à décomposer préalablement le mot-thème, et à s'inspirer de ses syllabes pour les idées qu'il allait émettre ou les expressions qu'il allait choisir. C'est sur les morceaux de l'anagramme, pris comme cadre et comme base, qu'on commençait le travail de composition (127).
} 
Tras algunas vacilaciones terminológicas (27-33; Wunderli, 1972b:42-54), producto de la voluntad de marcar la especificidad del fenómeno que había descrito, Saussure se decidió por anagrama como marbete genérico, precisando algunas de sus especies concretas con los nombres paragrama, hipograma, anafonía, paramorfo, locus princeps, maniquí, logograma, silabograma, etc. A pesar de la etimología del término y de su larga tradición poética y retórica - sobre todo renacentista y barroca, aunque también rastreable en época helenística-, Saussure entiende por anagrama un fenómeno esencialmente fónico $(27,31)$ de diseminación —más que de permutación, que es facultativa- de los dífonos o sílabas de un nombre en una porción textual más extensa (un sintagma o una frase, un verso o incluso una estrofa). No se trata de combinar las letras de un nombre en otro, sin resto posible (Alcuino y Luciano como anagramas de Calvino, Salvador Dalí anagramatizado en Avida Dollars), sino de extender las mallas fónicas de un nombre a un pequeño texto, convirtiendo a aquél en el bastidor sobre el que éste se teje. Saussure insistió siempre en la irreductibilidad de su anagrama a su homónimo más tradicional, que denominó «anagrama gráfico moderno» y calificó de «forma inferior» (72-73), así como a otras figuras como la paronomasia, la figura etimológica, el fonosimbolismo o la propia aliteración y la rima, que sí acercó en su momento al acoplamiento fónico.

Casi de inmediato, Saussure confirmó también la práctica de este anagrama en los versos saturnios, corpus de control sobre el que probaba todos sus descubrimientos. En ese punto dejó de interesarle el acoplamiento fónico, objeto hasta ese momento de lo que ahora denomina «vérification amusante», para centrarse en la búsqueda de esos segmentos fónicos que saturan el texto y promueven con ello su recolección en un nombre temáticamente pertinente. $Y$ así, en un verso del epitafio del Lucio Cornelio Escipión señalado arriba a propósito del acoplamiento, Saussure lee anagramatizado el nombre Scipio (29):

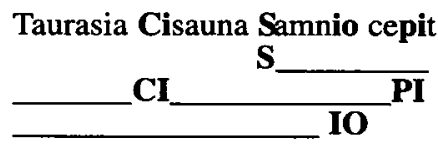

La búsqueda de esta poésie phonisante, y los sorprendentes hallazgos, se extendió a la poesía latina clásica, y en versos de Lucrecio, Virgilio, Horacio, Ovidio y Séneca, entre otros, descubre Saussure los nombres resonantes que los poetas habrían impuesto a su imaginación 
creadora verbal en el origen mismo de la composición. Así, una palabra-tema como Augustus haría resonar algunos de sus dífonos posibles, en su estricto orden además (lo cual no es preceptivo) en este verso de las Geórgicas (I, v.496): Aut gravibus rastris galeas pulsabit inaneS. En este caso el nombre es recuperable en el espacio de un solo verso, sin mediar insistencia, sobrerrepresentación de sus dífonos. En otros casos, en cambio, el nombre está diseminado en un fragmento de texto mayor, y entonces se hace necesaria esta reiteración de los dífonos conductores. Y así, en los primeros cincuenta hexámetros de De rerum natura Saussure descubre anagramatizado hasta diez veces el nombre de la diosa invocada en esos mismos versos, Venus, pero en su nombre griego, Aphrodité, más resonante que el latino, al decir de Saussure (79-100). Y así, por ejemplo, los versos 20-25, que contienen sin duda la más explícita invitación de Lucrecio a la diosa para que le acompañe en su tarea, para que ella, madre de todas las cosas vivientes, se alíe con él en su empeño de cantarlas, Saussure recupera dos veces el nombre $A p(h)$ rodité:

EFFICIS UT CUPIDE GENERATIM SAECLA PROPAGENT. QUAE QUONIAM RERUM NATURAM SOLA GUBERNAS, NEC SINE TE QUICQUAM DIAS IN LUMINIS ORAS

\section{DI}

A PRO

TE

EXORITUR, NEQUE FIT LAETUM NEQUE [AMABILE] QUICQUAM, TE SOCIAM STUDEO SCRIBENDIS VERSIBUS ESSE QUOS EGO DE RERUM NATURA PANGERE CONOR ${ }^{8}$

$\begin{array}{lllll}\text { OR>RO OD } & & \text { AP } & \text { [A } & \text { E] } \\ \text { TE } & \text { IT } & \text { IT } & & \\ \text { DI } & & \end{array}$

Las letras latinas fueron campo abonado para la proliferación del modelo compositivo anagramático, e incluso la prosa literaria -las cartas de Ausonio, Cicerón, hasta el mismo Julio César, la histo-

8 Les mots, pp.90-92. El abate Marchena tradujo así estos versos:

Pues como seas tú la soberana de la naturaleza, y por ti sola todos los seres ven la luz del día, y no hay sin ti contento ni belleza, vivamente deseo me acompañes en el poema que escribir intento de la naturaleza de las cosas. 
riografía de Valerio Máximo, etc.- exhibía esa misma inspiración verbal. Saussure llegó a afirmar que el anagrama debió ser una segunda naturaleza para todo romano educado que tomaba la pluma para decir hasta la cosa más insignificante (117), determinando casi exclusivamente la forma que el autor daba con sus palabras a su pensamiento (119-120) y sin perjuicio de los refinamientos que podía alcanzar en la obra de arte verbal. El maestro ginebrino prolongó, con éxito su investigación más allá del latín clásico y postclásico, hasta los humanistas del Renacimiento (Angelo Poliziano:139-146) y del Barroco (Thomas Johnson, un traductor al latín de epigramas griegos y Magister of Arts de Cambridge:146-148).

Y así, por ejemplo, en el epitafio de sólo ocho versos que Angelo Poliziano dedicó hacia 1480 a su amigo, el pintor Filippo Lippi, Saussure lee anagramatizados los nombres del autor, Politianus, del destinatario y su oficio, Philippus pictor, del mecenas de Lippi, Lorenzo de Medicis (Medices), todos ellos «tautológicos», es decir, enunciados literalmente en el propio texto, y el de la amante del pintor y hermana de sus asesinos, Leonora, anagrama en este caso excepcionalmente criptográfico. La breve extensión de la composición hace que los dífonos se solapen o incluso algunos sirvan para componer más de un nombre 9 .

Deseoso de hallar por fin una «prueba viva» de la secreta tradición del anagrama poético, llegó a escribir a un contemporáneo y colega de la Universidad de Bolonia, Giovanni Pascoli, autor de excelentes poemas latinos. Saussure, que analizó dichos poemas con resultados muy satisfactorios (149-151), le pregunta sin ambages en una de sus cartas si es de forma azarosa, o intencionadamente, que en algunos de sus versos, extraídos de dos pequeños poemas -Iugurtha (1896) y Catullocalvos (1897) — se reproduzcan las sílabas de ciertos nombres clave (Falerni, Ulixes, Circe y Iugurtha), en este caso en secuencias casi rigurosamente ordenadas:

..lfacundi calices hausere - alternil
FA AL GR AL ERNI
/Urbium simul/Undique pepulit lux umbras.resides
U UL U ULI X G S ES

9 Dice así el epitafio: Conditus hic ego sum picturae fama Philippus/Nulli ignota meae est gratia mira manus./Artifices potui digitis animare colores,/Sperataque animos fallere voce diu./Ipsa meis stupuit natura expressa figuris,/Meque suis fassa est artibus esse parem./Marmoreo tumulo Medices Laurentius hic me/Condidit; ante humili pulvere tectus eram. 


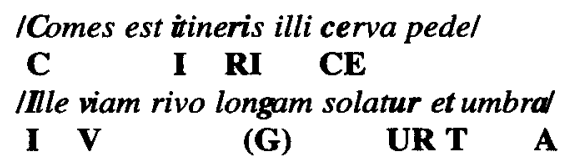

El maestro ginebrino tiene la prudencia de expresar a Pascoli sus dudas sobre «la realidad o fantasmagoría» (138) del fenómeno, pues es consciente de que «il y a quelque chose de décevant» en su investigación: «plus le nombre des exemples devient considérable, plus il y a lieu de penser que c'est le jeu naturel des chances sur les 24 lettres de l'alphabet qui doit produire ces coïncidences quasi régulièrement» (150-151; Nava, 1968:81). Confesándose además incapaz de abordar el cálculo de probabilidades a partir de la frecuencia estadística de los fonemas y de sus posibles combinaciones, que podría resolver el impasse, Saussure condiciona la prosecución de su investigación a una respuesta favorable del poeta. Por lo que sabemos, ésta no se produjo y el maestro ginebrino interrumpió en este punto su trabajo (Nava, 1968:75-78).

Sin duda debieron pesar en él, además, tanto la infructuosa búsqueda de una «prueba externa» de su hipótesis, es decir, de una alusión explícita al procedimiento en la tratadística de retórica, poética y métrica del pasado (134-137), como el escrúpulo por estar hurgando en el venero mismo de la inspiración de los clásicos, y por concluir que una ley severa pero - como afirma el propio Saussure- «déplorable en sa nature» (134) ordenaba la creación poética hasta en sus más mínimos detalles, de manera que «faire des vers avec anagramme est forcément faire des vers selon l'anagramme, sous la domination de l'anagramme» (30).

Además, incluso en los momentos de absoluta confianza en lo que llamó «la materialidad de los hechos» (133), una sombra se cernía sobre la viabilidad del fenómeno: la proposición de la causa que habría motivado el recurso al anagrama como «costumbre poética», como «viejo dispositivo indoeuropeo» transmitido a lo largo de un vasto periodo de tiempo y arraigado en literaturas muy dispares. Saussure se cuida mucho de proponer una sola (125), aludiendo, según el corpus textual objeto de sus análisis, bien a un principio estético derivado del equilibrio de los sonidos (126), bien a la idea superticiosa de que el nombre del dios védico debía estar diseminado en el texto para garantizar el éxito de la invocación (Benveniste, 1964:114), bien a razones de nemotécnica oral en el caso de la épica homérica. Pero sin duda fue 
consciente de la endeblez de una hipótesis que asumía al mismo tiempo que una ley rigurosa venía ordenando la creación poética durante más de dos mil años, y que esa tradición era oculta (133), una hipótesis que pretendía igualar bajo el mismo principio formal a una poesía sagrada, sujeta a un ritual, como la védica, y a la poesía latina del periodo de Augusto, laica y más lírica y subjetiva, sin hablar ya de la de los humanistas (125).

A pesar de todo, Saussure manifestó al final de su investigación la esperanza de que un día se viera explicado en su totalidad el código del que él sólo podía presentar «le maigre squelette» ${ }^{10}$, explicación que parece sumamente complicada en los términos estrictos propuestos por el maestro, relativos a la absoluta consciencia del método por parte del autor y del cómplice lector. Como veremos enseguida, la crítica de los Cuadernos de Anagramas ha puesto en evidencia además el escaso rigor de Saussure en el tratamiento de algunos textos, la modificación constante de los criterios y principios sentados con precedencia en virtud de «ligeras licencias transitorias» que acomodan el punto de vista a las concretas necesidades del análisis actual. No obstante, es razonable pensar que sus análisis no eran tan sólo delirios tocados de un impresionismo crítico poco atendible, sino que, como poco, reflejaban finas intuiciones sobre la combinatoria de las unidades fónicográficas y sobre el asociacionismo verbal.

\section{LA RECEPCIÓN DEL ANAGRAMA SAUSSURIANO}

\subsection{La vexata quaestio anagramática}

La publicación parcial de los inéditos saussurianos despertó el interés de la crítica ya desde las primeras entregas de Starobinski, lo cual se plasmó en una nutrida nómina de recensiones y comptes-rendus sobre todo en Francia en los primeros setenta (Rossi, 1968; Deguy,

10 Dice así Saussure (134): «Il arrivera un moment où l'on en ajoutera bien d'autres [règles] et où celles-ci paraîtront le maigre squelette du code dans son étendue réelle. On aura eu le temps, vu que nous n'en avons pris que l'essentiel, d'autre part de reconnaître que l'hypogramme en soi est tellement incontestable qu'il n'y a rien à redouter, ni pour son existence ni pour son exactitude, de la pluralité de voies qui s'ouvrent pour ses différentes réalisations». También en p.124. 
1969; Aron, 1970; Ronat, 1970; Wunderli, 1972c; Rey, 1973; Mounin, 1974; Meylakh, 1976 y Dupuis 1977). En 1974 tuvo lugar incluso una reunión internacional dedicada monográficamente a Les deux Saussure, cuyas actas fueron publicadas en dos revistas, tanto en francés como en inglés ${ }^{11}$. Casi todas las contribuciones consistían en una somera síntesis de la hipótesis de Saussure seguida de objeciones de distinto calibre y desde distintas perspectivas, las cuales, independientemente de su signo, consiguieron divulgarla, y a ellas se debe muy probablemente la acuñación de dos fórmulas que en lo sucesivo sirvieron de lema a dos actitudes hacia los Anagrammes contrapuestas y algo maniqueas: «la folie de Saussure» (Deguy, 1969) y «la seconde révolution saussurienne» (Aron, 1970). Esta situación no muy deseable tuvo su contrapunto de seriedad y lucidez en la magnífica monografía que dedicó Peter Wunderli (1972b) a la cuestión, en la que ofrecía un resumen de la hipótesis y mostraba su conexión con los temas centrales del Cours y su sorprendente presencia en algunas de las más influyentes poéticas contemporáneas.

Es de lamentar que el libro de Wunderli, escrito en alemán, fuera bastante desconocido, y que durante al menos una década la reflexión sobre estos textos saussurianos estuviera marcada por dos apriorismos críticos de signo contrario que, por su misma radicalidad, poco podían contribuir a perfilar un Saussure verdaderamente completo - y no absurdamente escindido en saberes y haceres incomunicados y hasta contradictorios- y a lograr una fértil asunción, con los ajustes debidos, de la hipótesis saussuriana en el panorama de la filología y la semiótica actuales. Dichas posiciones venían a coincidir a grandes rasgos con la contemporánea brecha establecida en Francia entre la ancienne y la nouvelle critique —la polémica Barthes-Picard-, aunque trasladada de medios teórico y crítico-literarios a medios semiolinguiísticos. Por un lado, algunos de los más conspicuos investigadores del legado del Cours, que señalaron la inconveniencia de dar a la luz pública unos papeles del sabio que eran en esencia un divertimento intrascendente, una boutade (Godel, 1960:6; Amacker, 1975:17; Engler, 1975:46) o bien una especulación demasiado osada para un mero diletante en cuestiones de teoría literaria y estética (Prosdocimi 1983: 36-38), y hasta criticaron acerbamente la labor de selección y

11 Recherches 16 (1974): Les deux Saussure y Semiotext(e) 2(1) (1975): Saussure's anagrams. Actas del Coloquio «The two Saussures» celebrado en la Universidad de Columbia el 12 y 13 de mayo de 1974, con trabajos de Bucher, Godzich, Irigaray, Lotringer, Pierssens, Riffaterre, y Starobinski. 
presentación de los textos por Starobinski (Mounin, 1974; Prosdocimi \& Marinetti, 1991:41) y la del segundo editor en importancia de los cuadernos, Peter Wunderli (Karlgren, 1973:97-98); por otro, aquellos que abogaban por una superación del paradigma estructuralista (Lacan, Kristeva, Barthes, Derrida, Baudrillard, Lyotard) y acogieron calurosamente la revelación de un Saussure que vendría a poner en entredicho algunos asertos centrales del Cours y de la lingüística estructural, como la arbitrariedad y la linealidad del signo linguístico, y que resultaría ser el Saussure más verdadero, frente al que malgré lui y acaso adulterando su pensamiento nos presentaron los editores y exégetas de los cursos de lingüística.

No vamos a ocuparnos aquí del rechazo displicente o de la flagrante omisión de los estudios sobre el anagrama poético en trabajos de conjunto sobre la obra saussuriana (además de los citados, Mounin, 1968 , Koerner, 1982), pues han sido sin duda superados por otras biografías intelectuales de Saussure más atentas a los Anagrammes (Culler, 1976, Gadet, 1987) ${ }^{12}$, así como por trabajos de lingüística saussuriana tan penetrantes como los de Peter Wunderli. Tampoco vamos a mostrar la inspiración anagramática de gran parte de las estrategias y corrientes de lo que podemos llamar, sin duda con cierta vaguedad, el postestructuralismo francés, pues daría para un estudio monográfico de considerable extensión ${ }^{13}$. Sólo apuntaremos aquí que hemos comprobado la presencia explícita o los ecos más o menos difusos de la teorización saussuriana tanto en los textos centrales de la desconstrucción (Derrida, 1978:94-95; 1975:241), como en las propuestas paragramáticas y semanalíticas de Kristeva (1978,I:227-269, II:55-216), como en momentos centrales del pensamiento crítico de Barthes (1986:121-122;1987:48), de Baudrillard (1981:51-52, 58; 1992:221-230, 250-252), de Lyotard (1979:267) y del último Lacan (1989:116). En una palabra, hemos decidido omitir aquí las referencias caracterizadas bien por su radicalidad en la adhesión o la censura, y que han perdido el brillo polémico ante otras más aquilatadas, bien por su recurso a los Anagramas más como provocador argumento de autoridad que con voluntad de acercarse a su contenido y discutir su posi-

12 Es significativo a este respecto el cambio de actitud de René Amacker, que en 1975 calificaba el anagrama de «absurdo pasatiempo» de Saussure, y en 1995 se convirtió en editor de la correspondencia Bally-Saussure, buena parte de la cual está consagrada, como sucede con la dirigida a Meillet, a la investigación anagramática, sobre la que Saussure tuvo puntualmente informados a sus discípulos más cercanos.

13 Hemos abordado tal estudio en dos capítulos extensos de nuestra tesis doctoral inédita. 
bilidad real. Son estas últimas las que aquí abordaremos, pero antes repasaremos brevemente - no es posible de otro modo- la presencia del anagrama en el panorama filológico hispánico en estas tres décadas transcurridas desde la exhumación de los cuadernos.

\subsection{El anagrama saussuriano en España}

Sorprende la casi total ausencia de referencias en España al trabajo saussuriano sobre los anagramas, habida cuenta de la repercusión que tuvo en medios filológicos del país vecino y su presencia e influencia sobre los trabajos de un maestro de lingüística y poética para muchas generaciones de filólogos españoles, Roman Jakobson, como mostraremos después. Ni siquiera estaba disponible en nuestra lengua la mayor parte de los textos saussurianos publicados, y durante varias décadas sólo hemos contado con la traducción de la primera edición parcial de Starobinski, la de 1964 (Nethol, 1977:229-247). Sólo muy recientemente ha aparecido la traducción Las palabras bajo las palabras (1996), novedad editorial que nos llega con veinticinco años de retraso, pero que puede sin duda acercar al lector hispánico la poética anagramática saussuriana. No obstante, es de lamentar que dicha traducción no sea la más adecuada y la que reclamaba una tan larga desatención ${ }^{14}$.

También en las últimas fechas ha aparecido un estudio dedicado monográficamente a presentar la hipótesis de Saussure, a relacionarla con conceptos clave de la semiótica del siglo XX como la iconicidad y la significancia y a aplicarla, con resultados de una sorprendente evidencia, a algunos poemas de Antonio Machado (Herrero, 1996). Dicha publicación puede contribuir, junto a los trabajos que nosotros hemos presentado recientemente y que abordan aspectos y desarrollos concretos de la hipótesis saussuriana (Rodríguez Ferrándiz, 1995, 1996), a divulgar y a suscitar nuevas aproximaciones al anagramatismo poético en el panorama filológico español.

14 En primer lugar, es objetable la decisión de ofrecer una pura y simple versión de Les mots sous les mots que carece tanto de un estudio introductorio o al menos una justificación editorial, como de la imprescindible actualización bibliográfica. En cuanto a la traducción en sí, peca en general de demasiado literal y, en ocasiones, es pedestre. En otras nos parece que comete errores de bulto: la expresión saussuriana factice (31), que quiere decir «facticia, artificial», es traducida por ficticia, y son otras varias las inexactitudes del mismo tenor. 
Aparte de esta reciente bibliografía hispánica sobre el anagrama, no tenemos noticia de la publicación de estudios críticos al respecto en el periodo transcurrido desde su revelación, ni siquiera de referencias en ediciones de obras de o sobre Saussure. Sólo nos constan alusiones al respecto de Fernando Lázaro Carreter en la introducción a sus Estudios de poética (1979 [1976]:10), de César Nicolás en el detallado análisis de un soneto de Manuel Machado (1983:181-201) y de Mario García-Page a propósito de una tipología de las figuras de dicción (1992:166). En cuanto a la incorporación a manuales de retórica hispánicos de los términos de la hipótesis saussuriana, sólo se ha producido muy recientemente, en las obras de Fernando Marcos Álvarez (1989), de José Antonio Mayoral (1994), de Joan Oriol Dauder y Joan Oriol i Giralt (1995) y de Helena Beristáin (1995), quienes, sin embargo, excepto en este último caso, no señalan la autoría del descubrimiento. Confiamos en que esta escuálida noticia del anagrama en nuestra linguiística y crítica literaria, junto a ese incipiente interés que acabamos de reseñar, justifiquen suficientemente estas páginas.

\subsection{El método saussuriano a examen}

Dos de las contribuciones críticas a la investigación saussuriana más atendibles - aun cuando no atañen principalmente a su aspecto más seductor (el anagrama en las letras latinas), sino al acoplamiento fónico en los versos saturnios y védicos- son las de Françoise Rastier y David Shepheard, que demuestran ser buenos conocedores tanto de los textos saussurianos como de las literaturas objeto del análisis del maestro. Dichos autores, trabajando independientemente, han llegado a conclusiones muy similares: Saussure maneja textos fragmentarios, que a menudo no delimitan la extensión de los versos singulares y sobre los que además varían notablemente las lecciones de unos editores a otros (es el caso de los saturnios) o textos muy probablemente interpolados y de lectura complicada debido a los efectos del sandhi o fonética sintáctica (en el caso del Rig-Veda). Dichas vacilaciones acerca de la literalidad de los textos desvirtúan los resultados de una investigación sobre el acoplamiento fónico en la que, como reconoció el propio Saussure, «pair o impair dépend d'une seule unité, et d'une seule erreur sur l'intention du versificateur» (26) y con la que se pretende describir un modelo compositivo en el que «pas un mot en pou- 
vait être changé ni déplacé sans troubler la plupart du temps plusieurs combinaisons nécessaires» (30). En el caso de los saturnios, Rastier apunta con toda razón que difícilmente era posible establecer de forma fehaciente la ley de acoplamiento y esa ley «subsidiaria y protectora» de la compensación en el verso siguiente cuando casi todos los que nos han llegado son versos sueltos pertenecientes a un dístico perdido, o dísticos que bien podrían integrarse en una composición más extensa y ya irrecuperable. Precisamente la dificultad de asignar al saturnio un modelo compositivo regular hace que cada editor establezca su propio corpus dependiendo de su particular caracterización métrica, y no de otro modo sucede con Saussure, que juzgará malos saturnios a aquellos que no se ajustan a su modelo fónico (Rastier, 1970:6-13,24). Por su parte, Shepheard demuestra que Saussure, apoyándose en las irregularidades métricas, síntomas de interpolaciones, y en el generoso margen que concede el sandhi, se permite eliminar estrofas del RigVeda que perturban el acoplamiento, o corregir el texto recibido de modo que surtiera los fonemas oportunos en cada caso (Shepheard, 1982:516-519). Es decir, Saussure abusó de la holgura que consentían unos textos establecidos sin demasiada fiabilidad, lo cual no invalida de hecho su hipótesis, pero la hace radicalmente inverificable al menos en lo referido a esos corpus poéticos concretos.

Si dejamos estas fases tempranas de la investigación y nos centramos en su núcleo más denso, referido al anagrama de un nombre propio en la poesía latina clásica y en los versificadores en latín de todos los tiempos, es claro que sorteamos el problema de la fiabilidad de los textos, pero otro de mayor calado subsiste. Se trata, sin duda, del flanco más débil de la hipótesis, aquél sobre el que más han insistido sus contradictores (Karlgren, 1973:100; Mounin, 1974:240-241; Dupuis, 1977:20-21; Prosdocimi \& Marinetti, 1990:62): si la extensión del material significante que presuntamente anagramatiza la palabra-tema es lo suficientemente grande (tampoco necesita serlo demasiado: unos pocos versos bastan), entonces es casi forzoso, por las repeticiones de un inventario muy limitado de fonemas, que sea posible recuperar ese nombre, lo que equivale a decir que cualquier nombre puede ser extraído de un texto de mediana extensión.

Es cierto que Saussure se esforzó en delimitar áreas anagramáticas restringidas (80-100), de extensión entre uno y seis versos, cuya textura fónica estaría saturada de los dífonos del nombre anagramatizado, así como marcas topológicas que acotaran esa inusual densidad fónica de ciertos fragmentos (el maniquí: una porción de texto, a veces un verso 
entero, cuyas letras inicial y final coincidían con la inicial y final de la palabra-tema: Priamides: Puppibus ignes 50-55; Aphrodite: Aeneadum genetrix hominum divomque 84). También señaló que el nombre anagramatizado habría de ser resonante, enjundioso, extraño en su silabismo, y no banal (Aphrodite y Priamides mejor que sus equivalentes Venus y Hector: 79-80,53). Pero es verdad que, una vez enunciados esos principios que harían verdaderamente significativas las repeticiones, se concedió generosas licencias en los casos más arduos: extendiendo el fragmento hasta más de seis versos, permitiendo el recurso a los monófonos (y no sólo dífonos) en la recomposición y admitiendo alteraciones de la cantidad vocálica, la permuta de ciertas consonantes y la evocación sólo parcial, y no completa, del nombre concernido. Algunos de los ejemplos allegados arriba pueden dar muestra de estas manipulaciones: Saussure admite el monofono $s$ para recomponer Scipio en el saturnio epigráfico que conmemora la muerte de Escipión el Africano, y se permite extraer el dífono -or-de exoritur y emplearlo, con inversión de orden, en la evocación de Aphrodite.

Saussure mismo fue consciente en varios momentos de su investigación de este delicado filo entre «la realidad y la fantasmagoría» del fenómeno:

\begin{abstract}
Une pièce n'offre que maigrement l'homogramme désiré: ainsi il est clair que nous nous berçons d'illusions, ou que nous voulons à toute force arracher au texte ce qu'il livre à peine. Une pièce offre surabondamment l'homogramme désiré: ainsi il est clair qu'on peut avoir partout quand on veut l'hypogramme désiré, que cela est une chose banale, inévitable par la somme des chances (131).

Quand un $1^{\text {er }}$ anagramme apparaît, il semble que ce soit la lumière. Puis

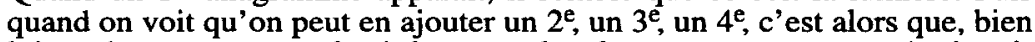
loin qu'on se sente soulagé de toutes les doutes, on commence à n'avoir plus même de confiance absolue dans le premier: parce qu'on arrive à se demander si on ne pourrait pas trouver en définitive tous les mots possibles dans chaque texte, ou à se demander jusqu'à quel point ceux qui se sont offerts sans qu'on les cherche, sont vraiment entourés de garanties caractéristiques, et impliquent une plus grande somme de coïncidences que celles du premier mot venu, ou de celui auquel on ne faisait pas attention (132).
\end{abstract}

El maestro llegó a practicar la verificación de una contraprueba en un cuaderno que llamó significativamente Contrôle, buscando - con relativo éxito- el nombre Pindarus en los primeros versos de la Eneida, que nada tiene que ver con el poeta griego (132). Por otro lado, un curioso y documentado lector de los cuadernos, Aldo Rossi, señaló que el nombre Leonora, que Saussure lee criptografiado en el 
epitafio de Poliziano al pintor Lippi ( $c f$. supra), no se corresponde con el de la amante del pintor, llamada Lucrezia..., nombre que el propio Rossi también extrae del texto incluso con mayor evidencia que el de Leonora (Rossi, 1968:119-122), pero dejando una seria duda sobre la fiabilidad de la escucha saussuriana.

En esta complicada tesitura de su hipótesis el maestro propuso, como recurso final, el cálculo de probabilidades de las recurrencias fónicas (131-132), con sus medias y sus desviaciones significativas, pero aseguró que dicho cálculo, complicado por «circonstances entourantes qui échappent à toute formule statistique» (Amacker, 1995:122), «défierait les forces des mathématiciens eux-mêmes» (132). Entonces, aún no había recurrido a esa «prueba viva» de consultar a un poeta anagramatista en activo, Pascoli, y sugirió que «la véritable pierre de touche est de recourir à ce que dira l'instinct d'une seconde personne non prévenue» (132). Pues bien, sólo un año después de la publicación por Starobinski de Les mots sous les mots vio la luz un trabajo que, de parte de persona no prevenida - al menos no alude nunca al Saussure de los Anagrammes-, aunque desde luego de manera no instintiva, sino apoyada por evidencias matemáticas, supone un muy razonable aval a la hipótesis del maestro ginebrino precisamente en ese aspecto tan frágil de la teoría como era la demostración fehaciente de la «matérialité des faits», es decir, de que los encuentros de los sonidos en los versos no eran azarosos, sino que, yendo más allá del umbral de expectativas de las repeticiones fónicas, mostraban una voluntad estética, armonizadora $\mathrm{y}$, acaso, también significativa.

\subsection{Las nuevas pruebas de la antigua poésie phonisante}

Nos referimos a la obra de Eva H. Guggenheimer, Rhyme Effects and Rhyming Figures. A Comparative Study of Sound Repetitions in the Classics with Emphasis on Latin Poetry, publicada en 1972. La autora sostiene que repeticiones fónicas de muy diverso tipo, como la rima de las palabras finales de los versos propia de la poesía romance, pero también otros efectos rítmicos como rimas internas, o ecos entre segmentos fónicos contenidos en palabras distintas y hasta la propia repetición de palabras enteras o de sintagmas en posiciones marcadas topológicamente, eran esquemas compositivos absolutamente convencionales en la poesía griega y latina clásicas. Con ello 
entiende rebatir la general creencia de que en la poesía clásica cuantitativa no existían efectos conscientes de repetición fónica y de que, cuando se producían, no se debían a una voluntad poética, sino que eran encuentros fortuitos causados por la flexión nominal o verbal, es decir, accidentes de la gramática y de la sintaxis ajenos al arte y a menudo desagradables para el oído antiguo. Por el contrario, la autora defiende y demuestra el alcance extraordinario de estas figuras de repetición fónica y la variedad de sus realizaciones, sometidas a rigurosos «sound patterns» que, según afirma categóricamente, «must have been considered deliberate» (1972:6), y establece una detallada tipología con numerosos ejemplos. El interés de este trabajo es extraordinario en orden a elucidar y asumir el desarrollado, más de seis décadas antes, por Saussure, acusado a menudo de impresionismo crítico, de alucinación proyectada por el afanoso analista sobre todo texto objeto de su auscultación: no sólo se ocupa en esencia del mismo fenómeno en los mismos corpus textuales - la poesía latina clásica como campo de pruebas principal, con verificaciones de apoyo en otros corpus poéticos- sino que además aborda, con resultados muy significativos, esa «prueba externa» fallida en Saussure, desvelando pasajes de Gorgias, Cicerón, Quintiliano, Martianus Capella y el autor de la Rhetorica ad Herennium, entre otros, que avalan la aguda conciencia de las armonías fónicas entre los clásicos.

El estudio de Guggenheimer plantea un espectro muy amplio de efectos fónicos posibles, minuciosamente clasificados en cuanto a la posición en los versos de los segmentos repetidos (inicial, final o no relevante) y al tamaño de éstos (monosílabos, bisílabos, palabras ligeramente modificadas, palabras idénticas, grupos de palabras, el verso o colon entero), lo cual descubre una preocupación por el rigor formal de las recurrencias fónicas que a veces echamos en falta - lo hemos apuntado arriba - en el trabajo de Saussure.

Es relativamente sencillo encontrar en la abundante relación de versos que recoge la autora fenómenos formalmente idénticos a algunos que conforman la hipótesis anagramática. Algunos son muy similares a los que Saussure mostraba como realizaciones del acoplamiento fónico, sobre todo cuando se producía por segmentos difónicos o mayores. Por ejemplo, Guggenheimer copia este verso: sanctius his animal mentisque capaciusaltae (Ovidio), en el que los segmentos ius, is y al del primer hemistiquio se duplican en el segundo (1972:128). El análisis de Guggenheimer es, incluso, más detallado que el saussuriano, pues a las repeticiones de los dífonos o trífonos dentro del 
mismo verso o en versos consecutivos se añade el paralelismo de las posiciones entre los hemistiquios y entre los versos. Valgan de ejemplo estos dos versos de las Geórgicas (IV, 371-372), que la autora copia separando los hemistiquios para evidenciar la simetría fónica vertical además de la horizontal. Copiamos los polífonos repetidos en el mismo verso a la derecha, y los repetidos entre verso y verso abajo (1972:140):

et gemina auratus

Eridanus, quo non alius taurino cornua vultu IN, AUR

per pinguia culta $E R, U S$

A ULT

La similitud con los pararelismos descubiertos por Saussure en los saturnios es evidente, como vimos arriba en los análisis del epitafio del cónsul Lucio Cornelio Escipión y del dístico de la epopeya de Livio Andrónico. De hecho, la ley del acoplamiento fónico saussuriana parece avalada por esta afirmación taxativa de Guggenheimer: «classical Latin poetry was composed according to patterns which involved the ordered repetition of sound effects» (1972:145). La aplicación sistemática de los patrones fónicos descritos por la autora a la épica griega y latina produce resultados tan significativos o más que a la lírica o a la elegía, hasta el punto de que todos «show continuous or recurring repetitions of sound in parallel metrical positions» (1972:149).

Los pasajes, según se indica, están elegidos aleatoriamente -casi como hacía Saussure en la etapa de mayor euforia anagramática- y algunos de ellos, como el primer canto de la Eneida, debido a su extensión, son analizados a partir de un programa de ordenador, refinamiento técnico que no estuvo al alcance del maestro pero que él auguró a la posteridad de los estudios anagramáticos. Según se desprende de estos análisis computerizados, la presencia regular de tramas fónicas más o menos densas delimita espacios de concordancias, lo que permite concluir que «the multiple repetitions not only create coherence and continuity but also are arranged in regular patterns. Frequent forms are couplets (a a), repetitions after one, two, or three intervening lines ( $\mathrm{a} \times \mathrm{a}, \mathrm{a} \times \mathbf{x} \mathrm{a}, \mathrm{a} \times \mathbf{x} \times \mathrm{a})$, and quatrains of various shapes ( $\mathrm{a} b$ b, a b a b, etc.)» (1972:149).

Ahora bien, hasta el momento el precedente saussuriano para las tesis de Guggenheimer se refiere a la versión inicial y «débil» del anagramatismo, la de los acoplamientos fónicos, mientras que la hipóte- 
sis saussuriana «fuerte», la del mot-thème anagramático, es mucho más ambiciosa, al pretender encontrar no sólo recurrencias y armonías fónicas, sino la alusión que con ellas se está haciendo a un nombre propio temáticamente relevante del texto. No obstante, la autora sostiene que ciertos temas fónicos concretos parecen estar, si no inseminando directamente todo el pasaje, sí construyendo su esqueleto fónico, su armazón o bastidor, lo cual está muy cerca de la hipótesis saussuriana. Así, por ejemplo, el análisis por ordenador aplicado a los finales de verso ${ }^{15}$ del primer libro de la Eneida demuestra la frecuencia extraordinaria de los «acoustic sets»-or- (como en oris, doloris, honores, -ent- (gentem, ventis, parentem)y -at- (penatis, Achatae, fatur). De un total de 776 versos, las terminaciones distintas son 205. Pues bien, de ellas 59 están formadas por el dífono -or-, 40 por el trífono -ent- y 28 por el dífono - at-, mientras que la frecuencia media de los otros 202 polífonos finales es de sólo 3'2 veces. Dichas frecuencias no están relacionadas ni con la distribución silábica más habitual en latín ni con una particular querencia idiolectal virgiliana: ninguno de los finales de verso de los setenta que conforman la Égloga VII contiene los dífonos -or-o -ent-. Guggenheimer reconoce que estas repeticiones de segmentos fónicos finales no parecen estar asociadas a un esquema periódico, a una alternancia fija, pero afirma que «their regularity can hardly be explained away as a result of accident» $y$ apunta que «further analysis will be required to determine the significance of the intervals in the end rhyme patterns of epic poetry» (1972:223). Análisis sobre textos de Catulo, Ovidio, Horacio y Lucrecio, en este caso realizados manualmente, confirman la presencia masiva también en ellos de determinadas - y distintas en cada caso- rimas armonizadoras del fragmento.

Abundando en este aspecto, Guggenheimer apunta, a partir de los datos comparativos de los primeros cantos de la Ilíada y de la Eneida, algo de una excepcional importancia para la formulación más radical de la hipótesis de Saussure: la centralidad del nombre del héroe protagonista en la armonización fónica de las rimas finales de verso. Así, en

15 Guggenheimer entiende por final de verso el segmento fónico formado por la vocal o el diptongo métricamente marcado y la consonante o consonantes que le siguen en el sexto pie del hexámetro, como fato y nati Esta condición mínima de la rima puede ser reforzada por otras correspondencias, como la identidad de la vocal final no marcada (fati y rati) y, en su caso, de las consonantes sucesivas, o la identidad del radical (fati y fatum). En cambio, no son tenidos en cuenta en el cómputo los casos en que los segmentos fónicos difieren en la segunda consonante, como en certo y cernis, arcus y arma. 
el primer canto de la Ilíada, el nombre Achilleus, en casos gramaticales distintos, comparece 17 veces en posición final de verso; el nombre Achaioi, 32 veces; el nombre Apollon, 13; y el nombre Agamemnon, 11. Guggenheimer demuestra que, en el caso de Achilleus, por ejemplo, su presencia parece atraer a su cercanía cotextual la serie de palabras etelle-alloi-ballei-metalla. Tanto es así que la autora señala, con valor de principio general:

\begin{abstract}
The formal quality of the ancient repertory of rhymes is most obvious if one compares rhymes and rhymes words in poems of different content and character. As a rule some rhyming figures, especially those involving proper names, but also repetitions of nouns and verbs, are intimately connected with the theme of the poem. The words used in these figures receive added emphasis from their prominent acoustic position and may properly by called «theme» words (1972:147).
\end{abstract}

No obstante, el principio no es aplicado en la práctica salvo en estos casos mayores citados, a diferencia de Saussure, que hizo de él, en cuanto fue atisbado, el motivo esencial de su hipótesis, aquél sobre el que giraron a partir de entonces toda la especulación y todos los ejemplos. En una fase todavía temprana de su estudio, Saussure lamentaba, una vez descubiertas «las homofonías poéticas» en Homero, «l'impossibilité de savoir quoi est imité ou quoi est imitant», pues, si no hay un nombre propio como catalizador de las recurrencias fónicas, «tout se borne à une valse des syllabes dans le cercle» (Amacker, 1995:119). Guggenheimer (1972:144) también encuentra el mismo escollo cuando señala que las múltiples repeticiones en un fragmento complican extraordinariamente la confección del diagrama de las correspondencias, pues los patrones de resonancias parecen solaparse o entrecruzarse, dificultando la proposición de regularidades.

Saussure, sin embargo, resolvió magistralmente está dificultad al subordinar todas las repeticiones fónicas dispersas a la evocación de un nombre, que sería la clave fono-temática del fragmento, condensando así tanto las expectativas semánticas del pasaje como los índices fónicos que alcanzan saliente textual debido tanto a su saturación como a su relevancia posicional. A pesar de que en sus esquemas y análisis Saussure parece preocupado casi exclusivamente por proveer los índices fónicos, por justificar la procedencia en el texto de todos y cada uno de los dífonos de la supuesta palabra-tema, insistió mucho sobre su peso temático, como vimos arriba. Es por ello por lo que, en una carta a Bally — un tanto reticente a asumir la posibilidad del ana- 
grama-, confesó que la cuestión capital no está en constatar que a partir de un solo verso pueden extraerse varios nombres, lo cual es evidentemente cierto, sino que en la mayoría de los casos es fácil extraer precisamente el que corresponde al «nom topique du passage» (Amacker, 1995:122). Queda, sin embargo, el espinoso tema de la consciencia absoluta del procedimiento que Saussure sostuvo siempre, y que le llevó a la encuesta al poeta anagramista, para él decisiva.

No hemos encontrado en toda la crítica de los Anagrammes a nuestra disposición alusiones a la capital obra de Guggenheimer, cuya importancia para la asunción del anagramatismo en su aspecto siquiera más técnico y material esperamos haber mostrado suficientemente. Se trata, sin lugar a dudas, del más serio espaldarazo al anagramatismo saussuriano desde la crítica literaria contemporánea, tanto más revelador cuanto desarrollado en ignorancia trabajo de Saussure y tanto más sorprendente cuanto aplicado sobre el mismo corpus textual analizado por Saussure.

\subsection{El anagrama en el análisis semiológico del texto poético: aportaciones y perspectivas}

A pesar de las pruebas favorables presentadas por Guggenheimer, la aplicabilidad del anagrama como estrategia general apta para el análisis semiológico del texto poético parece, sin embargo, muy complicada si nos apartamos de las letras clásicas, y sobre todo si pretendemos seguir al pie de la letra a Saussure en su convicción de que el procedimiento es consciente para el poeta y de que ordena la creación verbal hasta en sus más mínimos detalles. En tales términos, digámoslo ya, la hipótesis de Saussure es inviable. Desde nuestro punto de vista, se hace necesario tanto moderar el alcance textual del anagrama -que no es fórmula magistral del poema, sino figura retórica que ha de someterse a las condiciones de perceptibilidad y de satisfacción estética propias de toda figuralidad (Herrero, 1996:207) — como ampliar las posibilidades del método —que podrá escapar a la determinación consciente del poeta y tomar cuerpo bien como pulsión inconsciente, bien como inmanente estrategia que parte de la propia intentio operis, y es reconocida por una atenta intentio lectoris.

En cuanto a esta ampliación del método saussuriano, la crítica ha hecho interesantes propuestas, como no podría ser de otra manera, tras 
las aportaciones de las varias corrientes de inspiración psicoanalítica, de la estilística, de la psicosistemática, así como de aquellas otras más recientes que conceden la iniciativa interpretativa al lector, como la hermeneútica, la estética de la recepción, la desconstrucción o las varias teorías semióticas del lector ideal o modelo.

Jacques Lacan aseguró, tras conocer los Anagramas, que es en ese texto donde «Saussure espera a Freud» (1989:116) y Roman Jakobson ha reclamado la asunción de las estructuras linguíísticas latentes en la poesía en un trabajo en el que justamente recordaba la labor de pionero de Saussure (1973:280,282,287), citando unas palabras de los cuadernos en las que el maestro afirma con vehemencia que su anagrama no podía ser un juego accesorio de la versificación, sino su base más radical "que la critique d'une part, et que le versificateur d'autre part, le veuille ou non" (30). Con todo, es un hecho que a Saussure le pesó demasiado la dicotomía entre lo fortuito y lo premeditado, y probablemente ese tertium non datur condenó en buena medida la genial intuición de sus Anagramas. Starobinski lo dejó apuntado cuando afirmó:

Mais peut-être la seule erreur de Saussure est-elle d'avoir si nettement posé
l'alternative entre «effet de hasard» et «procédé conscient»? En l'occurren-
ce, pourquoi ne pas congédier aussi bien le hasard que la conscience?
Pourquoi en verrait-on pas dans l'anagramme un aspect du processus de la
parole, processus ni purement fortuit ni pleinement conscient (120).

Como oportunamente se cuestiona Jakobson (1973), «les mécanismes dégagés par l'analyse linguistique, ont-t-ils été visés délibérément et rationnellement dans le travail créateur du poète?», respondiendo que

\begin{abstract}
Un calcul des probabilités, aussi bien qu'une comparaison précise des textes poétiques avec d'autres sortes de messages verbaux démontrent que les particularités frappantes qui caractérisent la sélection, l'accumulation, la juxtaposition, la distribution, et l'exclusion, en poésie, des diverses classes phonologiques et grammaticales, ne peuvent pas être tenues pour des accidents négligeables régis par le seul hasard. Toute composition poétique significative, qu'elle résulte de l'improvisation ou soit le fruit d'un long et pénible travail, implique un choix orienté du matériel verbal (1973:280).
\end{abstract}

Jakobson (1973) señala que en muchos casos «la nature exacte des éléments pivots peut rester en dehors de la conscience de l'auteur», aunque tanto él como un «lecteur réceptif» son capaces de aprehender espontáneamente las ventajas estéticas de determinada elección, como 
se ha puesto de manifiesto a menudo al comparar el ante-texto de un poema y su versión definitiva (Jakobson, 1973:280, Lázaro Carreter, 1990:232-245).

El pensamiento semiótico de Jakobson no sólo quedó marcado por la enseñanza de Peirce, del que fue temprano lector y divulgador (Herrero, 1988:152-154,159-165; Abad, 1992:143-151), sino que estuvo «anagramáticamente orientado» no bien conoció la genial intuición de los cuadernos de Saussure. Jakobson acogió con gran entusiasmo la publicación de los extractos (1966:685; 1981:109-110; 1987:212-213), se convirtió en editor de algún texto clave en la investigación (1988:145-154) y puso en práctica de inmediato el anagrama como estrategia interpretativa de textos poéticos de la más variada procedencia, desde Dante a, Pessoa, pasando por Shakespeare y Baudelaire (1977:31-52; 99-123; 179-199; 235-260).

El ejemplo jakobsoniano, sin duda, motivó el interés no sólo de críticos literarios, sino también de lingüistas y semiólogos de variada orientación. Entre los primeros, Michael Riffaterre inspiró decididamente su semiótica de la poesía en el modelo saussuriano, que él llama primero paragramático (1979: 75-88) y después hipogramático (1983: 16-42 y 212), y modifica en alguna medida al conceder una mayor relevancia al aspecto temático de la diseminación que al estrictamente fónico. Geoffrey Hartman (1985: 137-154) también demostró conocer los textos saussurianos, aplicando el anagrama en sus análisis de poemas de Milton y Wordsworth; también, con declaración o no de la paternidad saussuriana, fue empleado en ensayos y análisis de críticos tan solventes como Oreste Macrí (1977: 3-75), Stefano Agosti (1972, 1982, 1983: 199-222) y Gian Luigi Beccaria (1975) y aplicado sobre poetas tanto del pasado (Petrarca, Sponde, Marino) como contemporáneos (Leopardi, Baudelaire, Valéry, Pascoli, Montale, Ungaretti). Y tanto Genette (1976: 69-70, 312) como Todorov (1972, 1978) se han hecho eco de los estudios saussurianos, el primero caracterizándolos como una etapa en la tradición secular del mimologismo, el segundo incluyéndolos en sus tipologías de teorías poéticas y de figuras retóricas.

Entre los lingüistas y semiólogos, quizá hayan sido François Rastier (1976: 110) y Katherine Kerbrat-Orecchioni (1977: 46-58) quienes más seriamente han analizado las posibilidades del anagrama como fenómeno textual, refiriéndolo a sus investigaciones en torno a las isotopías y a la connotación, respectivamente. $\mathrm{El}$ anagrama saussuriano ha encontrado también una sanción poderosa, aunque de momento 
sólo implícita, tanto en la linguiística del texto como en la psicosistemática de estirpe guillaumiana. La lingüística del texto, inspirada sin duda en la magistral lección greimasiana (1973:161-176), ha puesto en el origen teórico de todo texto un tópico o tematextual entendido como la estructura semántica nuclear que lo condensa y a menudo sustanciado en una figura nominal relativamente constrictiva, lo cual supone una confirmación de la hipótesis saussuriana desde el lado del significado (García Berrio y Albaladejo, 1983 y Albaladejo, 1984), y al tiempo revela la intuición de Saussure al promover el ámbito de sus análisis anagramáticos a las relaciones discursivas, y no meramente frásticas. Y la psicosistemática, por su parte, ha puesto en el centro del interés lingüístico lo que llama «la raison du signifiant», la significancia como cruce absolutamente habitual, y por ello precisamente ajeno tanto a la conciencia del hablante como a la taxonomización lingüística, de las connotaciones semióticas y de las connotaciones semánticas que generan las palabras en nuestro discurso efectivo. Dicho cruce a menudo es textualizado como proyección fonotemática que orienta el avance del propio discurso, como quería la palabra-tema saussuriana, pero además es capaz de explicar satisfactoriamente muy variados fenómenos de linguiística tanto sincrónica como diacrónica (ChevalierLaunay-Molho, 1984; Launay, 1986).

Pero la más decidida propuesta que avala el interés teórico de los anagramas, sin duda, ha partido de la llamada «lingüística de la escritura». Uno de sus más destacados representantes, Jonathan Culler (1989: 185187), se refería en una reciente ponencia programática a los Anagramas como la referencia de la nueva lingüística, y afirmaba:

\begin{abstract}
No sólo no nos apercibimos de la mayoría de las reglas y regularidades de nuestra lengua, sino que todos hemos tenido la experiencia de ver un texto iluminado por patrones y resonancias que no habíamos advertido previamente, pero que, una vez que se nos han hecho patentes, parecen del todo inevitables. El problema resaltado por la búsqueda de anagramas por parte de Saussure es la exclusión que una lingüística del signo y el código hace de una serie de patrones potenciales, de los que es difícil determinar si tienen o no efectos de significación. Sus ejemplos problemáticos permiten vislumbrar la posibilidad de que lo que llamamos códigos, fenómenos discretos y signos o sistemas de signos son sólo casos especiales de una interminable iteración generalizada o formación de patrones (Culler, 1989:187).
\end{abstract}

Como se aprecia, la cuestión anagramática, que quedó abierta en la formulación saussuriana, sigue todavía abierta y suscita interesantes aproximaciones desde los más variados ámbitos de la reflexión filológica y 
semiótica. No juzgamos exagerado afirmar que los Cuadernos de Anagramas y su fértil posteridad deben contarse entre las más deslumbrantes teorías del lenguaje figurado de nuestro siglo, y hacen de Saussure, además de maestro de lingüística, maestro también de poética.

Aquí hemos presentado la hipótesis y la crítica, y hemos debido necesariamente omitir aspectos que juzgamos cruciales, como la caracterización retórica del anagrama poético y su posible inclusión en las tipologías de figuras al uso, la presencia en los cuadernos de nociones que anticipan o traslucen las empleadas en los cursos de linguíística general y la condición esencial de nombre propio de la palabra anagramatizada, dimensiones inexploradas en las que trabajamos en estos momentos y que deberán completarse con la imprescindible aportación de ejemplos poéticos que evidencien en el análisis la figura anagramática en sus diversas expresiones.

\section{Referencia bibliográficas}

Abad Nebot, F. (1992). «Peirce, Jakobson y la esencia de la literatura y del lenguaje». Signa 1, 143-151.

Agosti, S. (1972). Il testo poetico. Teoria e pratica d'analisi. Milano: Rizzoli.

- (1982). Cinque analisi. Il testo della poesia. Milano: Feltrinelli.

- (1983). «Funzioni anagrammatiche e rappresentazioni verbali nella poesia barocca: da Sponde e da Marino». En Il segno barocco. Testo e metafora d'una civiltà, G. Nocera (ed.), 199-222. Roma: Bulzoni.

Albaladejo, T. (1984). «Estructura de sentido, representación textual semántico-intensional y tópico textual». Anales de la Universidad de Murcia XLIII: 1-2, 265-284.

AMACKER, R. (1975). Linguistique saussurienne. Génève: Droz.

- (1995). «Correspondence Bally-Saussure», CFS 48, 91-134.

ARON, Th. (1970). «Une seconde révolution saussurienne?». Langue française 7, 56-62.

AvalLE, D.S. (1973). «La sémiologie de la narrativité chez Saussure». En Essais de la théorie du texte, Ch. Bouazis y otros (eds.), 17-49. París: Galilée.

BARTHES, R. (1986). Lo obvio y lo obtuso. Barcelona: Paidós.

- (1987). El susurro del lenguaje. Más allá de la palabra y de la escritura. Barcelona: Paidós.

BAUDRILLARD, J. (1981). De la seducción. Madrid, Cátedra.

- (1992). El intercambio simbólico y la muerte. Caracas: Monte Ávila.

BECCARIA, G. L. (1975). L'autonomia del significante. Figure del ritmo e della sintassi. Torino: Einaudi. 
BenVENiste, É (1964). «Lettres de Ferdinand de Saussure à Antoine Meillet». CFS 21, 89-130.

BerISTÁIN, H. (1995). Diccionario de retórica y poética. México: Porrúa.

CULleR, J. (1976). Saussure. Sussex: Harvester.

- (1989). «Hacia una lingüistica de la escritura». En La lingüística de la escritura, N. Fabb y otros (eds.) 181-192. Madrid: Visor.

CheValier, J.C., M. LaUnAY, M. MolHo (1984). «La raison du signifiant». Modèles linguistiques VI: 2, 27-41.

DeguY, M. (1969). «La folie de Saussure». Critique 260, 20-26.

DERRIDA, J. (1975). La diseminación. Madrid: Fundamentos. - (1978). De la gramatología. México: Siglo XXI.

DuPUIs, M. (1977). «À propos des anagrammes saussuriennes». Cahiers d'Analyse textuelle 19, 7-24.

ENGLER, R. (1975). «Sémiologies saussuriennes: 1. De l'existence du signe». CFS 29, 45-73.

GADET, F. (1987). Saussure. Une science de la langue. París: P.U.F.

GarCía BerRio, A. y T. AlBALADEJo (1983). «Estructura composicional Macroestructuras». Estudios de Lingüistica de la Universidad de Alicante 1, 127-179.

GARCÍA-PAGE SÁNCHEZ, M. (1992). «Datos para una tipología de la paronomasia» Epos 8, 155-243.

GenetTe, G. (1976). Mimologiques. Voyage en Cratylie. París: Seuil.

GODEL, R. (1957). Les sources manuscrites du Cours de linguistique générale de F. de Saussure. Genève: Droz [1969²].

- (1960). «Inventaire des manuscrits de Ferdinand de Saussure remis à la Bibliotèque publique et universitaire de Génève». CFS 17, 5-11.

GugGENHEIMER, E.H. (1972). Rhyme Effects and Rhyming Figures. A Comparative Study of Sound Repetitions in the Classics with Emphasis on Latin Poetry. La Haya-París: Mouton.

Hartman, G. (1985). Easy Pieces. New York: Columbia U.P.

Herrero Blanco, A. (1988). Semiótica y creatividad. La lógica abductiva.

Madrid: Palas Atenea.

- (1996). «La "sibilación escrita". Anagramatismo en la poesía de Antonio Machado». Bulletin Hispanique 98: 1, 205-219.

JAKOBSON, R. (1966). Selected Writings IV. La Haya-París: Mouton.

- (1973). Questions de poétique. París: Seuil.

- (1977). Ensayos de poética. México: F.C.E.

- (1981). Lingüística, poética, tiempo. Conversaciones con Krystina Pomorska Barcelona: Crítica.

- (1988). «La primera carta de Ferdinand de Saussure a Antoine Meillet sobre los anagramas». En Obras Selectas, I. Madrid: Gredos.

JAKOBSON, R. y L. WAUGH (1987). La forma sonora de la lengua. México: Siglo XXI.

KARLGREN, H. (1973). «Ferdinand de Saussure and his anagrams». Statistical Methods in Linguistics, 91-100.

Kerbrat-OreCChIONI, C. (1977). La connotation. Lyon: P. U. de Lyon. 
KoERNER, E.F.K. (1982). Ferdinand de Saussure. Madrid: Gredos.

KrISTEVA, J. (1978). Semiótica. Madrid: Fundamentos, 2 vols.

LACAN, J. (1989). El Seminario de Jacques Lacan. Libro 20: Aun (1972-3).

Buenos Aires: Paidós.

LAUNAY, M. (1986). «Effet de sens: produit de quoi?». Langages 82.

LÁzARo CARreter, F. (1976). Estudios de poética. Madrid: Taurus.

- (1990). De poética y poéticas. Madrid: Cátedra.

Lotringer, S. (1974). «Le 'complexe' de Saussure». En Recherches 16, Les deux Saussure, 90-111.

LyotaRd, J. F. (1979). Discurso, Figura. Barcelona: Gustavo Gili.

MACRÍ, O. (1977). «L'angelo nero e il demonismo nella poesia montaliana». En Due saggi, 3-75. Lecce: Milella.

Marcos Állarez, F. (1989). Diccionario práctico de recursos expresivos (figuras y tropos). Cáceres: Universidad de Extremadura.

MAYORAL, J. A. Figuras retóricas. Madrid: Síntesis.

MeYLAKH, M. (1976). «À propos des anagrammes». L'Homme XVI: 4, 105115.

MINASSIAN, M. (1976). «Sur la correspondence de Meillet avec Saussure relative aux anagrammes». BSL, 351-359.

Mounin, G. (1968). Saussure ou le structuraliste dans le savoir. París. Seghers.

- (1974). «Les anagrammes de Saussure». En Studi saussuriani per Robert Godel, R. Amacker, T. de Mauro y L.J. Prieto,(eds.), 235241. Bologna: Il Mulino.

NAVA, G. (1968). «Lettres de Ferdinand de Saussure à Giovanni Pascoli». CFS $24,73-81$.

Nethol, A. M. (ed.) (1977). «Los anagramas de Ferdinand de Saussure (textos inéditos)». En Ferdinand de Saussure. Fuentes manuscritas y estudios, 229-247. México: Siglo XXI.

NiCOLÁs, C. (1983): «Del fonema al contexto: sobre un soneto de Manuel Machado» Cuadernos de la Cátedra Miguel de Unamuno 27/28, 181-201.

OrIOL DAUDER, J.A. i J. ORIOL i GIRALT. (1995). Diccionari de figures retòriques $i$ altres recurs expressius. Barcelona: Llibres de l'Índex.

OssolA, C. (1979). «"Attestazione" e "sottoscrittura": gli ipogrammi di Saussure». Il piccolo Hans 22, 6-43.

Prosdocimi, A. (1983). «Sul Saussure delle leggende germaniche». CFS 37, 35-106.

Prosdocimi, A. y A. MarinetTi (1991). «Saussure e il saturnio. Tra scienza, biografia e storiografia». CFS 44, 37-71.

RASTIER, François (1976). «Sistemática de las isotopías». En Ensayos de semiótica poética A. J. GreIMAS y otros (eds.), 107-140. Barcelona: Planeta.

— (1970). «À propos du Saturnien». Latomus XXIX: 1, 3-24. Recherches 16, (1974).

REY, J.M. (1973). «Saussure avec Freud». Critique 309, 136-167. 
RIFFATERRE, M. (1979). La production du texte. París: Seuil.

- (1983). Sémiotique de la poésie. París: Seuil.

RODRÍGUEZ FERRÁNDIZ, R. (1996). «Del anagrama al emblema: la publicidad de los nombres». En Investigaciones Semióticas VI. Actas del VI Congreso Internacional de la Asociación Española de Semiótica: "Mundos de ficción», II, 1349-1360. Murcia: Universidad de Murcia, 1996.

- (En prensa). «El mito del signo y el signo del mito en Saussure y en Lotman». Comunicación leída en la Reunión Internacional in memoriam Iuri M.Lotman. Granada, 26-28 de octubre de 1995.

RoNAT, M. (1970). «Vers une lecture des anagrammes par la théorie saussurienne». Change 6, 119-126.

Rossi, A. (1968). «Gli anagrammi di Saussure: Poliziano, Bach, Pascoli». Paragone 218, 113-127.

SAussure, F. de (1978). «Essai pour réduire les mots du grec, du latin et de l'allemand à un petit nombre de racines» (Introducción de Boyd Davis). CFS 32, 73-101.

- (1983). Curso de lingüística general [Traducción, prólogo y notas de Amado Alonso. Edición crítica de Tullio de Mauro]. Madrid: Alianza Editorial.

- (1986). Le leggende germaniche. [Ed. A. Marinett y M. Meli]. Este: Zielo.

SHEPHEARD, D. (1982): «Saussure's Vedic Anagrams». Modern Language Review 77: 3, 513-523.

- (1984). «Saussures Anagramme und die deutsche Dichtung». Sprachwissenschaft 11: 1-2, 52-79.

STAROBINSKI, J. (1971). Les mots sous les mots. Les anagrammes de Ferdinand de Saussure. París: Gallimard. [Trad. esp. Las palabras bajo las palabras. La teoría de los anagramas de Ferdinand de Saussure. Barcelona: Gedisa, 1996].

- (1974). «Deux cahiers inédits sur Virgile» Recherches 16. Les deux Saussure, 113-146.

TOdorov, T. (1972). «Les sens des sons». Poétique 11, 446-462.

- (1978). Les genres du discours. París: Seuil.

- (1981). Teorías del símbolo. Caracas: Monte Ávila.

WuNDERLI, P. (1972a). «Ferdinand de Saussure: “1 ${ }^{\text {er }}$ cahier à lire préliminairement" Ein Basistext seiner Anagrammstudien». Zeitschrift für Französische Sprache und Literatur LXXXII :3, 193-216.

- (1972b). Ferdinand de Saussure und die Anagramme. Tübingen: MaxNiemeyer.

- (1972c). «Saussure et les anagrammes». Travaux de Linguistique et Littérature 10, 1, 35-53. 